\title{
Influence of an introgression from Triticum timopheevii into chromosome 5A of bread wheat cultivars Saratovskaya 29 and Diamant 2 on agronomical, physiological and biochemical parameters under contrasting irrigation conditions
}

\author{
Simonov A.V. ${ }^{1 *}$, Smirnova O.G. ${ }^{1}$, Permyakov A.V. ${ }^{2}$, Permyakova M.D. ${ }^{2}$, \\ Osipova S.V. ${ }^{2}$, Pshenichnikova T.A. ${ }^{1}$ \\ ${ }^{1}$ Institute of Cytology and Genetics, SB RAS, Novosibirsk, Russia \\ ${ }^{2}$ Siberian Institute of Plant Physiology and Biochemistry, Irkutsk, Russia \\ *email: sialexander@bionet.nsc.ru
}

The relative species enlarge a variability of bread wheat. From the species Triticum timopheevii, a line 821 inherited an introgression in the subtelomeric region of the long arm of 5A chromosome flanked by the markers Xgwm179 and Xgwm291. It includes the gene for leaf pubescence $H l^{t t}$ determining the formation of long, rare trichomes. Leaf pubescence plays an adaptive role in the response to abiotic stresses by changing the processes of gas exchange. The drought-tolerant cultivar Saratovskaya 29 (S29) carries two pubescence genes that form a dense layer of short trichomes. The non-droughtresistant cultivar Diamant 2 (Dm2) is practically glabrous. The aim of this work was to assess the effect of introgression carrying the gene $H l^{t t}$ on productivity and physiological and biochemical parameters under contrasting irrigation conditions. For this, substitution lines S29(821-5A) and Dm2(821-5A) were created. Under drought and irrigation conditions, the line $S 29(821-5 \mathrm{~A})$ showed a reduced productivity in comparison with the recipient: the number of grains per plant by $17-19 \%$ and the weight of 1000 grains by 15-28\%. The line Dm2(821-5A) retained the yield under irrigation and the weight of 1000 grains increased by $9 \%$. Under drought, this line surpassed the recipient for weight of 1000 grains by 1.5 times. These differences can be explained by the differences in physiological properties. In the line Dm2(821-5A), under both conditions, a decrease in transpiration rate and stomatal conductance was noted, and the efficiency of water use doubled. In S29(821-5A), under both conditions the photosynthetic parameters did not change. Under both conditions, S29(821-5A) showed a decrease of the total activity of the main antioxidant enzymes. Thus, the described introgression is favorable for Dm2, but negatively affects the drought tolerance of S29.

Acknowledgements: The work was carried out within the budget project No. 0259-20210018. 